\title{
AN ABSOLUTE DETERMINATION OF THE ${ }^{235} \mathrm{U}$ FISSION CROSS SECTION AT $964 \mathrm{keV}^{*}$
}

\author{
D. M. Gilliam $\dagger$ and G. F. KNoll \\ The University of Michigan, Ann Arbor
}

(Received 18 December 1974)

\begin{abstract}
An absolute measurement of the ${ }^{235} \mathrm{U}$ fission cross section has been carried out using a ${ }^{24} \mathrm{Na}-\mathrm{Be}$ photoneutron source with median neutron energy of $964 \mathrm{keV}$. A symmetric two-foil experiment was set up to measure the fission rate in a low-albedo laboratory, and variations in the sourceto-foil spacing used to determine the room background. Fission fragments passing through a limited solid angle aperture were recorded from each foil by solid state tracketch techniques. The photoneutron source was calibrated after each run using the manganese bath method and the secondary national standard source NBS-II. A computed neutron source spectrum with $32 \mathrm{keV}$ FWHM was derived by the Monte Carlo method and used in reducing the data to a cross section at $964 \mathrm{keV}$. The final value of $1.21 \pm 0.025$ barns is absolute in that, except for small corrections, its determination was independent of any other cross section data.
\end{abstract}

\section{INTRODUCTION}

The need for improved neutron-nuclear cross section data in the $\mathrm{keV}$ energy range is well recognized, the primary motivation being improvement of the data for fast breeder reactor calculations. The present measurement at $964 \mathrm{keV}$ was undertaken with the objective of contributing to the establishment of a more accurate absolute cross section standard in an energy range of substantial technological interest.

Although several critical assemblies and prototype fast breeders have recently come into operation and have provided much valuable macroscopic data for reactivity and criticality calculations, the need for reliable microscopic cross section data persists, especially for three purposes: (i) calculation of doubling times, (ii) calculation of performance of advanced alternative fuels, and (iii) calculation of reactor performance in accident situations.

Since the ${ }^{235} \mathrm{U}$ fission cross section has been widely employed as a standard by which other cross sections are measured, it is important that the ${ }^{235} \mathrm{U}$ fission cross section be established to very high accuracy, at least $1 \cdot 0-2 \cdot 0 \%$. Within the past ten years at least six other laboratories have reported measurements of this cross section, but the quoted errors of the results were generally $2.5 \%$ or larger. The present result has an estimated accuracy of $2 \cdot 1 \%$.

* Sponsored by the U.S. Energy Research and Development Administration.

$\dagger$ Present address: National Bureau of Standards, Washington, D.C. 20234.
In the establishment of a standard cross section, systematic errors can be detected and reduced by utilization of two or more independent experimental methods wherever possible. While most precise fission cross section experiments have employed fission chambers or gas scintillators, the fission counting in the present measurement was done by the completely independent track-etch method.

\section{EXPERIMENTAL METHOD}

In general, three basic quantities must be determined to establish a reaction cross section absolutely:

(i) the reaction rate,

(ii) the average neutron flux, and

(iii) the number of target nuclei.

In the present work, the corresponding quantities actually measured were

(i) the number of fission tracks recorded on polyester films, the geometry of the limited solid angle apertures, and the duration of the exposure;

(ii) the photoneutron source emission rate relative to NBS-II (the widely-intercalibrated, secondary standard source of the National Bureau of Standards) and the source-target geometry; and

(iii) the mass of the $\mathrm{U}_{3} \mathrm{O}_{8}$ deposits.

\subsection{An overview of the experimental procedure}

Three major runs were made: two symmetric, dual-detector runs as illustrated in Fig. 1, with source-detector spacings of $5.1 \mathrm{~cm}$ and $9.2 \mathrm{~cm}$, respectively; and one single-detector run with a source-detector spacing of $21.0 \mathrm{~cm}$. In this section, 


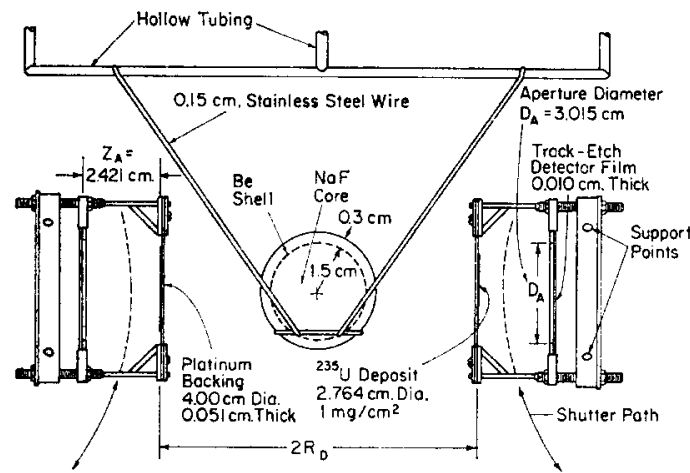

Fig. 1. Detectors and photoneutron source. The detectors and source holder (shown here) along with the shutter mechanism and tubular supporting structures are enclosed in a helium-filled drum $60 \mathrm{~cm}$ dia by $60 \mathrm{~cm}$ high, with walls of $\mathrm{Cd}$ sheet $0.025 \mathrm{~cm}$ thick. The tracketch detector material is (du Pont "Cronar") polyester film.

the experimental procedure for a typical run is outlined.

The $\mathrm{Na}-\mathrm{Be}$ photoneutron source is rotated slowly during activation at the centre of one face of a ( $2 \mathrm{MW}$ pool-type) reactor to insure uniform activation of the sodium. About $30 \mathrm{Ci}$ of ${ }^{24} \mathrm{Na}$ activity is produced, leading to an initial neutron yield of about $3 \times 10^{7} \mathrm{sec}^{-1}$. The source is then transferred to a support ring between the dual fission detectors as shown in Fig. 1. The source, detectors, and lightweight supporting structures are enclosed in a helium-filled drum $60 \mathrm{~cm}$ in diameter by $60 \mathrm{~cm}$ high, with walls of thin $\mathrm{Cd}$ sheet $(0.025 \mathrm{~cm}$ thick).

In each of the identical detectors, fission events are counted by collection of fission fragments on a polyester track-etch detector film through about $14 \%$ of $2 \pi$ solid angle. Electrically actuated shutters define the period of track accumulation $(45 \mathrm{hr})$. During the track collection period, the sourcedetector package is suspended at the centre of a large shielded cell (Fig. 2) having a $5 \mathrm{~cm}$ thick borax lining on the walls to reduce back-scatter of slow neutrons from the concrete. The spacing of the uranium deposit and the corresponding detector aperture is about $2.4 \mathrm{~cm}$, less than half the range of the heaviest fission fragment in the helium atmosphere which surrounds the source-detector structure. Following the fission track accumulation, the source is removed from the shielded cell while the manganese bath is filled and allowed to reach thermal equilibrium. Then the neutron source is transferred back into the cell and inserted quickly into the manganese bath (Fig. 3) for a carefully timed interval of about thirty hours. After removal of the

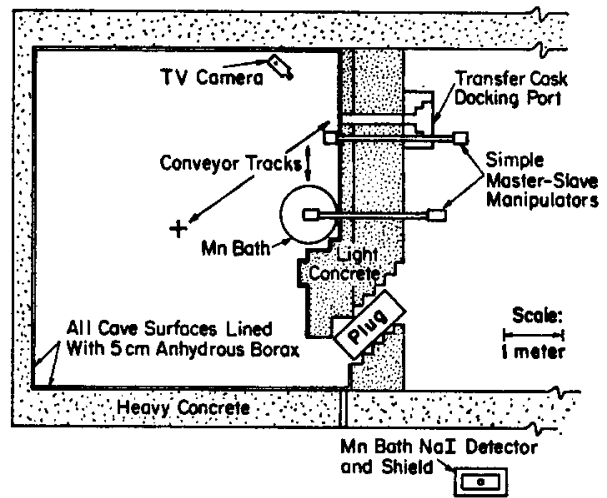

Fig. 2. Laboratory configuration. The mean radius of the shielded-cell is $2 \cdot 1 \mathrm{~m}$. The manganese bath is drained during the track accumulation period to maintain the full advantage of the low-albedo walls.

$\mathrm{Na}-\mathrm{Be}$ source and the decay of the bath activity to near zero, the reference source NBS-II is inserted into the bath to determine the bath efficiency. While the neutron source calibrations are in progress, the track-etch films are retrieved, etched, and mounted for counting on a projection microscope. At least two independent counts are made of each film, covering the entire exposed area, and with records kept of the individual subtotals on each of the $1 \mathrm{~mm}^{2}$ subdivisions of the films. The two independent counts of the 714 individual subdivisions of each film are compared for consistency, discrepancies are examined, and total counts are recorded.

The two fission foils for this experiment were analyzed for isotopic content and total $\mathrm{U}_{3} \mathrm{O}_{8}$ mass by the supplier, the Isotope Target Laboratory at Oak Ridge National Laboratory.

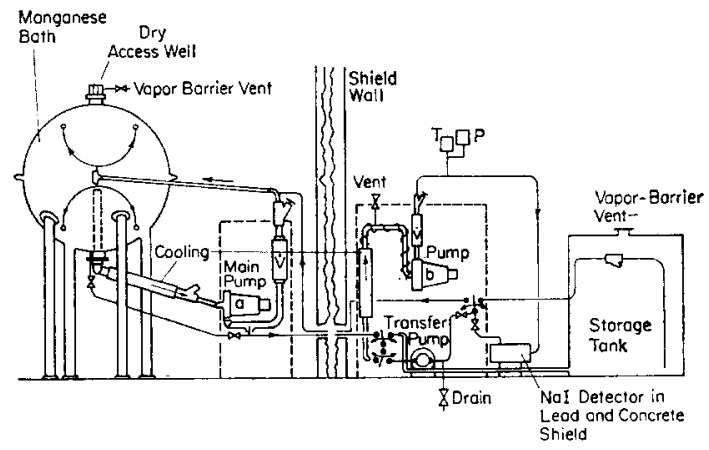

Fig. 3. The manganese bath neutron source comparator. The diameter of the sphere is $93.6 \mathrm{~cm}$. Flow meters are indicated with the label $\dot{V}$. The labels $T$ and $P$ mark the position of temperature and pressure sensors. All $\mathrm{Na}-\mathrm{Be}$ source calibrations for this cross section measurement were made by direct comparison to NBS-II on loan from the U.S. National Bureau of Standards. 


\subsection{Experimental details}

Fission counting. The latent tracks were developed by heavy etching to form round or oval pits of about $14 \mu \mathrm{m}$ dia. The etching solution was $6.25 \mathrm{~N} \mathrm{KOH}$ at $33^{\circ} \mathrm{C}$. Figure 4 shows the distribution of minor diameters of the etched pits. In addition to the fission fragment pit distribution (the major peak) there is a minor peak due to the presence of small background pits. The density and size distribution of the background pits was found to be the same on both front and back surfaces of the film. The consistency of repeated counts was much improved by beginning each count with a track size distribution measurement and specifying a minimum acceptable diameter in the valley between the two peaks. The use of circle templates and the projection microscope display made it possible to make track size distribution measurements rapidly. A square grid with $1 \mathrm{~mm}$ spacing and $3 \mu \mathrm{m}$ line width was ruled directly onto the pitted surface of the detector film in order to organize the total area into subdivisions which would fit within the field of view of the projection microscope. In counting the tracks within a given $1 \mathrm{~mm}^{2}$ square, a hand tally was used to add up the tracks as they were circled with a marker on the projection microscope screen. The interior count and edge-intersecting counts for

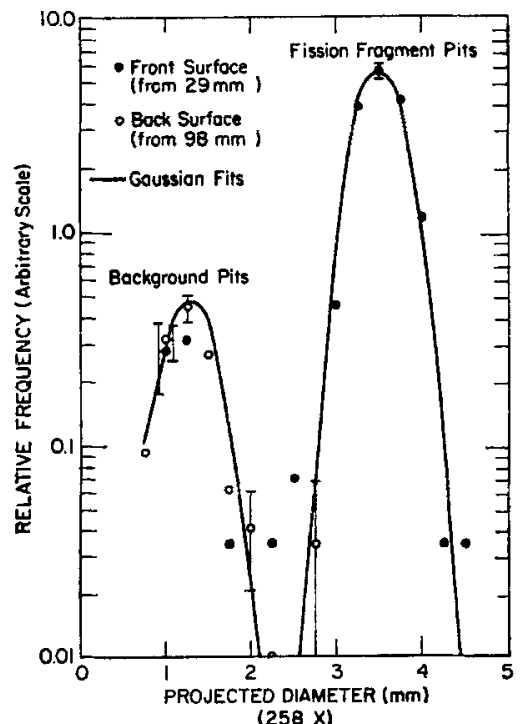

Fig. 4. Typical track size distribution. All of the tracks were etched heavily enough to be of circular or oval shape. The track (minor) diameters were measured with a circle template on the projection microscope screen. A counting threshold was set in the valley between the background pit distribution and the fission fragment pit distribution. each $1 \mathrm{~mm}$ square were recorded and mapped for both counts of every sample.

Because the detector efficiency is a sensitive function of the distance between the uranium target and the defining aperture, special pains were taken to minimize the error from this geometric factor. By means of an arrangement of gage blocks and $0.001 \mathrm{in}$. dial indicators, reproducibilities of better than $0.0006 \mathrm{~cm}(0.0003 \mathrm{in}$.) were obtained in repeated measurements of the deposit-aperture spacing. An uncertainty of $0.0025 \mathrm{~cm}$ was assumed in this spacing, however, because of deviations from perfectly flat, parallel geometry. The aperture diameter was determined within $\pm 0.002 \mathrm{~cm}(0.0008 \mathrm{in}$.) by means of a metallurgical microscope with 0.0001 in. stage micrometers.

Manganese bath. The manganese bath source comparator (Fig. 3) is continuously stirred and sampled by recirculation of the solution, with a portion of the recirculated liquid passing through a shielded detector chamber surrounding a $\mathrm{NaI}$ scintillation detector. The source is placed at the bath centre in a thin-walled dry well. An inward radial flow pattern prevents development of large stagnant eddies. The recirculation replaces the entire solution volume every $5 \mathrm{~min}$ and provides rapid turbulent mixing. The rapidity of the mixing was verified by injection of small activated volumes of solution into the bath at various radii. It was found that activity introduced at any point in the sphere was homogenized after 9 min to the extent that the concentration at the detector had converged to within $0.5 \%$ of the final value. Flow rates were found to fluctuate less than $2 \%$, and the temperature at the detector was held within $1^{\circ} \mathrm{F}$ of the set point $95 \cdot 5^{\circ} \mathrm{F}$.

Extremely stable, low-noise electronics, a specially selected photomultiplier tube (gain insensitive to count rate), and a gain-insensitive counting window ( $50 \mathrm{keV}$ to $2.50 \mathrm{MeV}$ ) combined to give very nearly constant neutron detection efficiency.

A correction for photoneutron production by the natural deuterium content of the aqueous manganous sulfate solution was determined by direct measurement. The saturated bath activity was observed following insertion of a ${ }^{24} \mathrm{Na}$ source similar in activity and physical construction to the photoneutron source, but lacking the beryllium shell. The relative gamma activities of the ${ }^{24} \mathrm{Na}$ source and the ${ }^{24} \mathrm{Na}-\mathrm{Be}$ sources were compared by means of a $\mathrm{Ge}(\mathrm{Li})$ detector. The constancy of the $\mathrm{Ge}(\mathrm{Li})$ active volume and the reproducibility of the geometric positioning were verified by repeated counts with a radium reference source. 
The neutron flux in the source position of the dry well was measured by the cadmium difference method to allow accurate correction for neutron absorption by the dry well structure and by the source itself.

The fission foils. The enriched uranium oxide deposits were vacuum-evaporated onto platinum foils and fired in air at $800^{\circ} \mathrm{C}$ to insure complete conversion of the oxide to the single stoichiometric form $\mathrm{U}_{3} \mathrm{O}_{8}$. The masses of the final oxide deposits were determined from microbalance weighings to be $8.16 \mathrm{mg}$ and $6.85 \mathrm{mg}$, giving mass thicknesses of the order of $1 \mathrm{mg} / \mathrm{cm}^{2}$. The isotopic composition of the deposits was (in atom per cent) ${ }^{235} \mathrm{U}, 99.432 \%$; ${ }^{234} \mathrm{U}, 0.040 \%$; ${ }^{236} \mathrm{U}, 0.471 \%$; and ${ }^{238} \mathrm{U}, 0.057 \%$.

The distribution of uranium over the deposit area was determined by alpha scanning with a coarse aperture $(2.5 \mathrm{~mm} \mathrm{dia})$. The foils were found to have a smooth gradient of $\mathrm{U}_{3} \mathrm{O}_{8}$ thickness amounting to about $12 \%$ from one edge of the deposit to the other. (This measured variation in thickness was taken into account in the computations of the detector efficiency and the average scalar flux.)

\section{DATA ANALYSIS, CORRECTIONS AND RESIDUAL ERRORS}

Derivation of the cross section from the measurements described above is straightforward and requires few corrections exceeding $1 \%$.

\subsection{The fission counts}

The total fission counts are obtained from the track counts and the calculated detector efficiency.

In addition to the imprecision always associated with Poisson distributed counts, several other types of errors must be evaluated in estimating the accuracy of the track counts. There is an uncertainty of about $1 \%$ in separating the lower portion of the fission track distribution from the upper portion of the background pit distribution (see Fig. 4). The reproducibility of the counts by a given scanner was found to be about $0.5 \%$ by comparison of repeated counts. This reproducibility error results primarily from judgment inconsistencies in applying the shape, size and depth criteria for track acceptance in borderline cases. An uncertainty of $0.5 \%$ due to scanner bias is assumed, based on the average difference in repeated counts of the same sample by different scanners. Finally, unresolvable coincidences in the locations of the tracks require a very small correction and associated uncertainty $(0.24 \%$ or less on any single film). Combining all sources of error, the total counts on the pair of detector films were $23,535 \pm 1.33 \%$ at $R_{D}=5.1 \mathrm{~cm}$ and
$11,623 \pm 1 \cdot 80 \%$ at $R_{D}=9 \cdot 2 \mathrm{~cm}$. For the singlefoil measurement (using the $8.16 \mathrm{mg}$ deposit) at $R_{D}=21.0 \mathrm{~cm}$, the track count was $1527 \pm 2.85 \%$.

Tests reported by the authors (1970) showed that the track-etch method gives $100 \% \frac{+0 \%}{-1 \%}$ registration efficiency for limited solid angle accumulation even through a mass-thickness of helium gas similar to that used in the present experiment. In these tests it was also noted that the aperture efficiency was the same with the collimator evacuated as when filled with helium. Furthermore, the region of $100 \%$ track registration for heavy ions shown by Benton and Nix (1969) should comfortably include all fission fragments even if their range is half-spent. On the basis of these considerations, $100 \% \pm 0 \%$ registration was assumed, and no residual error contribution has been included for net in-scatter or out-scatter by the helium gas.

A computer code to calculate the efficiency of the detector was written with provisions for including the significant anisotropy of fragment emission, the measured uranium thickness distribution, and the computed scalar flux variation over the deposit area. The fragment emission parallel to the neutron path is $(11.4 \pm 1.2) \%$ higher than at $90^{\circ}$ to the neutron path, according to measurements by Nesterov (1965). The functional form of the angular dependence assumed in the code was the empirical correlation found by Nesterov: $W(\theta) \mathrm{d} \Omega \propto(1+$ $\left.A \cos ^{2} \theta\right) \mathrm{d} \Omega$, where $\theta$ is the angle in the laboratory coordinates. The computed detector efficiency is $(5.08 \pm 0.91) \%$ higher than the corresponding value for isotropic emission. The estimate of the error in this adjustment is taken to be the full value of the difference in the adjustment as derived from two separate anisotropy measurements: that of Nesterov (1965) and that of Simmons and Henkel (1960). The correct normalization of the $W(\theta)$ function must account for the momentum of the compound nucleus and the slight subsequent forward bias in the angular distribution. The emission into the forward $2 \pi$ solid angle was found to be $1.0049 \pm 0.0012$ fragments per fission by means of a simple kinematics calculation based on average fragment mass values. The uncertainties in the measurements of the aperture diameter and deposit-aperture spacing (see Section 2.2) lead to residual errors in the detector efficiency of only $\pm 0.13 \%$ and $\pm 0.14 \%$, respectively. The non-uniformity of the uranium oxide thickness and the neutron flux variation over the target area were found to have only very small influence on the detector efficiency, resulting in insignificant residual errors. 
Table 1.

\begin{tabular}{|c|c|c|c|}
\hline $\begin{array}{c}\text { Source-Detector } \\
\text { Spacing, R } \\
\text { (cm) }\end{array}$ & Type of Perturbation & $\begin{array}{l}\text { Correction } \\
\text { to the } \\
\text { Fissiont } \\
\text { Count } \\
(\%)\end{array}$ & $\begin{array}{l}\text { Estimated } \\
\text { Residual } \\
\text { Error } \\
\text { (\%) }\end{array}$ \\
\hline $5.1,9.2$ & $\begin{array}{l}\text { Scanner Consistency (For the } \\
\text { sum of two beparate films) }\end{array}$ & & 0.35 \\
\hline 21.0 & $\begin{array}{l}\text { Scanner Consistency (For a } \\
\text { single film) }\end{array}$ & & 0.50 \\
\hline Any & Scanner Bias & & 0.50 \\
\hline 5.I & $\begin{array}{l}\text { Counting Statistics } \\
\text { Background Pit Discrimination } \\
\text { Track overlap }\end{array}$ & 0.16 & $\begin{array}{l}0.65 \\
0.98 \\
0.16\end{array}$ \\
\hline 9.2 & $\begin{array}{l}\text { Counting Statistícs } \\
\text { Background Pit Discrinination } \\
\text { Track Overlap }\end{array}$ & 0.03 & $\begin{array}{l}0.93 \\
1.38 \\
0.03\end{array}$ \\
\hline 21.0 & $\begin{array}{l}\text { Counting Statistics } \\
\text { Background Pft Discrimination } \\
\text { Track Overlap }\end{array}$ & 0.00 & $\begin{array}{l}2.56 \\
1.00 \\
0.00\end{array}$ \\
\hline Any & Fragment Enission Anisotropy & 5.08 & 0.91 \\
\hline Any & $\begin{array}{l}\text { Angular Distribution Normali- } \\
\text { zation in Laboratory Frame }\end{array}$ & 0.49 & 0.12 \\
\hline Any & Aperture-Deposit Spacing & & 0.14 \\
\hline Any & Aperture Oiameter & & 0.13 \\
\hline
\end{tabular}

In Table 1 , the correction factors and residual errors associated with the determination of the total fission count are summarized.

\subsection{The average scalar flux}

The determination of the flux from the manganese bath data and source-detector spacing measurements involves several adjustments and corrections.

Figure 5 shows the time development of ${ }^{56} \mathrm{Mn}$ activity in the manganese bath following insertion of a neutron source at $t=0$ for a period $T$. The observed ${ }^{56} \mathrm{Mn}$ activity is recorded from each $600 \mathrm{sec}$. counting interval during the entire activation and decay cycle. A saturated activity value (referred to

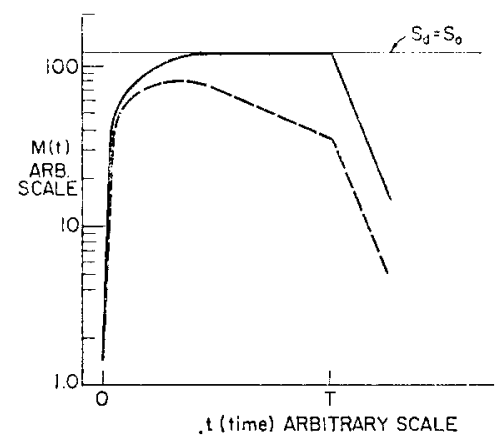

Fig. 5. Typical manganese activity histories for a constant source (solid line) and an exponentially decaying $\mathrm{Na}-\mathrm{Be}$ source (dashed line). The initial activity of the two sources is the same, $S_{d}=S_{0}$. The source in both cases was inserted into the bath centre at $t=0$ and removed at $t=T$. the time of source insertion) is computed from each individual counting interval using published values for ${ }^{56} \mathrm{Mn}$ and ${ }^{24} \mathrm{Na}$ half-lives (Merritt, 1971; Emery, 1972). Corrections for finite mixing time, background and deadtime are included in the computation of the saturated activity values. The half-life values and mixing behaviour have been sufficiently well determined that the adjusted saturated activity values show no deviation-other than statistical variation-over the entire $35-\mathrm{hr}$ activation-decay cycle. The weighted average of the saturated activity over the entire cycle has a statistical precision of about $0.03 \%$, and repeated counts with the same reference source showed an efficiency reproducibility of better than $0.1 \%$ over a period of a year.

Small corrections must also be applied to the manganese bath results to account for parasitic absorption of neutrons in the source and walls of the dry well, for neutron escape by streaming from the dry well or by bulk penetration, and for photoneutron production caused by the natural deuterium content of the aqueous solution.

As a basis for corrections for parasitic absorption, thermal flux measurements were made in the dry well for the case of the NBS-II source. The agediffusion equations of Wallace and LeCaine (1943) and measurements by Ryves (1964) were used to calculate the dry well flux for both the $\mathrm{Na}$-Be source and for the Ra- $\gamma$-Be source. The age-to-thermal was taken to be $6.7 \mathrm{~cm}^{2}$ for the $\mathrm{Ra}-\gamma-\mathrm{Be}$ neutrons and $14.9 \mathrm{~cm}^{2}$ for the $\mathrm{Na}-\mathrm{Be}$ neutrons. The calculated dry well flux for NBS-II agreed well with the measured value $\left(0.011 \mathrm{~cm}^{-2} \mathrm{sec}^{-1}\right.$ per unit source strength). The parasitic absorption correction for the Na-Be source was based entirely on the calculated dry well flux $\left(0.005 \mathrm{~cm}^{-2} \mathrm{sec}^{-1}\right.$ per unit source strength). The neutron streaming from the dry well was calculated from the effective solid angle of the opening with consideration given to streaming of the slow neutrons returning to the cavity as well as the direct streaming from the source. A conservative upper bound on bulk penetration escape of $0.34 \%$ was established for the $964 \mathrm{keV}$ neutrons by comparison with leakage measurements on ${ }^{252} \mathrm{Cf}$ neutrons made by DeVolpi (1971). Bulk penetration leakage for the lower energy $\mathrm{Ra}-\gamma-\mathrm{Be}$ neutrons from NBS-II is negligible. The correction for photoneutron emission by the natural deuterium content of the aqueous manganese sulfate solution was determined experimentally to be $(0.94 \pm 0.10) \%$ as discussed in Section 2.2.

The neutron emission rate of NBS-II was taken to be $1.174 \times 10^{6} \mathrm{sec}^{-1} \pm 0.5 \%$ (referred to June, 
1972), based on independent calibrations by four laboratories: the National Bureau of Standards (Noyce, 1963; Spiegel, 1972), the International Bureau of Weights and Measures (Huynh, 1970), the National Physical Laboratory (Axton, 1965) and Argonne National Laboratory (DeVolpi, 1969). The respective values reported by NBS, BIPM, NPL and ANL were $1.176,1 \cdot 177 \pm 0.003,1.168$ and $1.174 \pm 0.004$ in units of $10^{6} \mathrm{sec}^{-1}$ referred to June, 1972. The square root of the variance of these four values is $0.31 \%$. The comparable uncertainties associated with the NBS and NPL measurements were not stated explicitly. Axton quoted an accuracy of $\pm 0.2 \%$ in the manganese-to-hydrogen absorption ratio, random error of $\pm 0.25 \%$ and other systematic errors of less than $\pm 0.3 \%$ for the NPL calibrations. Noyce (1963) stated an uncertainty of $1 \cdot 1 \%$ for the NBS-I calibration, but the result of his method of error combination is not directly comparable with the one-standard-deviation errors used in the present work. Noyce's $1.1 \%$ represents the (linear) sum of the random error and $\frac{2}{3}$ of the (linear) sum of the systematic error components. If all the error components listed by Noyce were added in quadrature, the resulting combined error for the NBS calibration would be $0.62 \%$. The present error estimate $(0.5 \%)$ is equal to the maximum deviation from the mean value of the four equally weighted results.

It should be understood that the absolute calibration of the standard neutron sources is not dependent on an assumed value for the manganese capture cross section. The ratio of capture in hydrogen to capture in manganese must be known, but this ratio is usually determined directly by extrapolation of the count rates induced by a long-lived neutron source as the manganese/hydrogen concentration of the solution is changed.

A summary of the major corrections and residual errors in the manganese bath source comparisons is given in Table 2. The error associated with uncertainty in the ${ }^{24} \mathrm{Na}$ half-life is discussed separately in Section 3.4.

The determination of the scalar flux is completed by calculation of the flux per unit source strength, based on the source-detector geometry measurements. Corrections for scattering within the source, from the platinum backing of the deposits, and from nearby structural components are necessary.

A Monte Carlo code developed by Bensch and Vesely (1969) was employed to calculate the neutron and gamma transport within the photoneutron source. The calculated energy distribution is discussed in Section 3.4. The calculated angular
Table 2.

\begin{tabular}{|c|c|c|}
\hline Type of Perturbation & $\begin{array}{l}\text { Correction to } \\
\text { Na-Be/NBS-II } \\
\text { Neutron Yield } \\
\text { Ratio ( }(\bar{s})\end{array}$ & $\begin{array}{c}\text { Estimated Residuml } \\
\text { Uncertainty in the Ratio } \\
\text { (\%) }\end{array}$ \\
\hline $\begin{array}{l}D(Y, n) \text { Reaction } \\
\text { in Solution }\end{array}$ & 0.94 & 0.10 \\
\hline Parasitic Absorption & 0.63 & 0.36 \\
\hline Kixing Delay & 0.18 & 0.05 \\
\hline $\begin{array}{l}\text { Neutron Streaming } \\
\text { and Penetration }\end{array}$ & 0.16 & 0.18 \\
\hline $\begin{array}{l}\text { Count1ng Statistics, } \\
\text { Galn Varlations, } \\
\text { Background, Dead } \\
\text { Time, Ho Half-11fe } \\
\text { Errors }\end{array}$ & --- & 0.05 \\
\hline NBS-II Calibration & --- & 0.50 \\
\hline
\end{tabular}

distribution of neutron emission was taken into account in computing the average scalar flux acting on the fission deposits at the various source-detector spacings. The spherical shell geometry of the beryllium gives rise to a peaked angular distribution in which the peaking corresponds to angles of maximum chord length through the shell. At the closest source-detector spacing $(5.1 \mathrm{~cm})$ the offradial peaking in the angular distribution results in a scalar flux of about $3 \%$ higher intensity than would be realized from a point source of the same emission rate at the same spacing.

One factor not included in the Vesely code is the anisotropy of the photonuclear reaction. To assess the magnitude of the effect of this anisotropy on the scalar flux, some limiting cases were investigated by means of simple streaming calculations. The following data of Corman (1964) were used: $\mathrm{d} \sigma_{y, n} / \mathrm{d} \Omega=a+b \sin ^{2} \theta_{1}$, where $a / b=1 \cdot 2$ and $\theta_{1}=$ the angle between the velocity vectors of the neutron and the gamma ray. The non-trivial $\sin ^{2} \theta_{1}$ term tends to accentuate the off-radial peak in the angular distribution, but leads to only a small increase $(0 \cdot 12 \%)$ in the scalar flux intensity even at the closest source-detector spacing $(5 \cdot 1 \mathrm{~cm})$.

In this dual detector experiment the cross section is derived from the sum of the flux on the two detectors and the combined fission rate. The obvious advantage of this method is that it is significantly easier to get an accurate value for the sum of the fluxes on the two detectors than for the individual fluxes, because the sum is less sensitive to the exact placement of the source between the detectors. The distance between the detectors must still be measured carefully, but this step can be done in the absence of the source. Thus, a precise measurement of the location of the highly radioactive source is avoided. To illustrate, the estimated error in the measurement 
of the detector spacing, $\pm 0.008 \mathrm{~cm}$ for the $10 \mathrm{~cm}$ detector separation, leads to an uncertainty of $0.16 \%$ in the sum of the fluxes, while the estimated error in the source centreing, $\pm 0.050 \mathrm{~cm}$, leads to an uncertainty of only $0.28 \%$ in the sum of the fluxes.

The measured non-uniform uranium distribution on the deposits was taken into account in computing the sum of the fluxes, that is, the weighted average scalar flux over the target nuclei on the two deposits.

A correction of $(1.67 \pm 0.21) \%$ was required to account for scattering in the platinum backings (of $0.051 \mathrm{~cm}$ thickness). This correction was calculated by means of a second Monte Carlo program. In-scatter to the deposit at angles near the deposit plane was found to override out-scatter so that the net effect was to increase the scalar flux. The anisotropy of scattering from platinum was taken into account in this computation.

Scatter from nearby structural components increased the flux at the detector deposits by $(0.33 \pm$ $0 \cdot 17) \%$.

In Table 3 a summary is given of the major corrections and residual errors in the derivation of the average scalar flux per unit source yield.

\subsection{The number of target nuclei}

The masses of the $\mathrm{U}_{3} \mathrm{O}_{8}$ deposits were determined by microbalance weighings in an inert atmosphere at regulated pressure. The Isotope Target Laboratory at ORNL estimates that these weighings are accurate to $\pm 2 \mu \mathrm{g}$. However, since factors other than microbalance calibration and technique must be considered, a more conservative accuracy of $\pm 30 \mu \mathrm{g}$ has been assumed in this error analysis. For example, hot platinum surfaces can adsorb contaminants of $1-2 \mu \mathrm{g} / \mathrm{cm}^{2}$ thickness without any

Table 3.

\begin{tabular}{|c|c|c|c|}
\hline $\begin{array}{l}\text { Source-Detector } \\
\text { Spacing, } \mathrm{K}_{\mathrm{D}} \\
\text { (cai) }\end{array}$ & Type of Perturbation & $\begin{array}{l}\text { Adjustments and } \\
\text { orrections to the } \\
\text { Scalar Flux } \\
\text { (\%) }\end{array}$ & $\begin{array}{c}\text { Estimated } \\
\text { Restdual } \\
\text { Uncertainty } \\
(\mathrm{z})\end{array}$ \\
\hline 5.1 & Off-Radial Angular Flux Peaks & 3.09 & 0.40 \\
\hline 9.2 & Off-Radial Angular Flux Peaks & 0.79 & 0.20 \\
\hline 21.0 & Off-Radial Angular Flus Peaks & 0.20 & 0.10 \\
\hline 5.1 & Posttioning Uncertalnties & - & 0.32 \\
\hline 9.2 & Positioning Uncertainties & --- & 0.16 \\
\hline 21.0 & Positioning Uncertainties & $\cdots$ & 0.25 \\
\hline Any & Scattering in Pt Backings & 1.67 & 0.21 \\
\hline $5.1,9.2$ & $\begin{array}{l}\text { Scattering from other } \\
\text { Structural Components }\end{array}$ & 0.33 & 0.17 \\
\hline 21.0 & $\begin{array}{l}\text { Scattering from other } \\
\text { Structural components }\end{array}$ & 0.33 & 0.40 \\
\hline
\end{tabular}

perceptible change in appearance. In comparing gravimetric analysis with alpha assay and destructive coulometric analysis, White (1965) found that an accuracy of $30 \mu \mathrm{g}$ could be achieved with fission foils very similar to those used in the present experiment. Several studies of $\mathrm{UO}_{2}-\mathrm{U}_{3} \mathrm{O}_{8}$ stoichiometry indicate that no discrepancies in the $\mathrm{U}_{3} \mathrm{O}_{8}$ mass of more than $0.1 \%$ should be expected in the present case due to incomplete conversion of the oxide to the $\mathrm{U}_{3} \mathrm{O}_{8}$ form (Rodriguez de Sastre et al., 1967; Scott and Harrison, 1963; Peakall and Antill, 1960). Finally, an allowance of $0 \cdot 1 \%$ uncertainty is made for chemical impurities in the material supplied for these deposits. The combined uncertainties lead to an estimated $\pm 0.50 \%$ accuracy in the $\mathrm{U}_{3} \mathrm{O}_{8}$ masses.

A correction of $(0.15 \pm 0.04) \%$ was made to the effective ${ }^{235} U$ mass to account for the fission contribution of other uranium isotopes.

\subsection{Spectrum-averaged cross section and the normalization to $964 \mathrm{keV}$}

Two final steps in the data analysis remain to be explained. A correction must be made for the contribution of the room-scattered neutrons to the observed fission counts, and finally a correction is applied to derive a cross section value at $964 \mathrm{keV}$ from the observed neutron spectrum-averaged cross section, $\bar{\sigma}$.

The total observed fission count can be represented as the sum of two terms: the first due to fissions caused by the direct neutron flux streaming from the source and the second due to fissions caused by the room-scattered flux. For a single detector experiment the expression for the total track count $N_{F}$ is

$$
N_{F}=\epsilon \bar{\sigma} N^{25} \bar{\phi} T_{E}+\epsilon_{w} \bar{\sigma}_{w} N^{25} \phi_{w} T_{E}
$$

where $\epsilon$ is the detector efficiency, $\bar{\sigma}$ is the fission cross section averaged over the $(\gamma, n)$ source spectrum, $N^{25}$ is the number of ${ }^{235} \mathrm{U}$ nuclei, $\bar{\phi}$ is the photoneutron flux averaged over the deposit surface and exposure time $T_{E}, \epsilon_{w}$ is the (slightly different) efficiency for detection of fissions induced by the room-scattered flux $\phi_{w}$, and $\bar{\sigma}_{w}$ is the (much larger) average fission cross section for the room-scattered flux which has a degraded energy spectrum. In a large room with the source at the centre, the roomscattered flux is very nearly constant over small displacements in the neighbourhood of the source, while the photoneutron flux $\bar{\phi}$ drops off approximately as $1 / R_{D}{ }^{2}$. The qualification "approximately" must be included because of the angular distribution 
of the source emission and the finite extent of the fission deposit. At any source-detector spacing $\left(R_{D}\right)$, one can solve (1) for the apparent cross section, $\bar{\sigma}_{A}$ :

$$
\begin{aligned}
\bar{\sigma}_{A} & \equiv N_{F} / \epsilon N^{25} \bar{\phi} T_{E} \\
& =\bar{\sigma}+\epsilon_{w} \bar{\sigma}_{w} \phi_{w} / \epsilon \bar{\phi}
\end{aligned}
$$

The term $\epsilon_{w} \bar{\sigma}_{w} \phi_{w} / \epsilon \bar{\phi}$ is approximately proportional to $R_{D}{ }^{2}$ or precisely proportional to an appropriately defined "effective $R_{D}{ }^{2}$." A plot of $\bar{\sigma}_{A}$ vs $1 / \epsilon \bar{\phi}$ (or the effective $R_{D}{ }^{2}$ ) is a straight line with intercept $\bar{\sigma}$.

Equations (1) and (2) may be generalized in a straightforward way to describe the present dual detector experiment and to account for the differences in source strength in the different runs. Using the subscripts I and II to denote the two separate detectors, Equation 1 becomes:

$$
\begin{aligned}
\frac{N_{F \mathrm{I}}+N_{F \mathrm{I}}}{S}= & \bar{\sigma}\left(\epsilon_{\mathrm{I}} N_{\mathrm{I}}^{25} F_{\mathrm{I}}+\epsilon_{\mathrm{II}} N_{\mathrm{II}}{ }^{25} F_{\mathrm{II}}\right) \\
& +F_{w} \bar{\sigma}_{w}\left(\epsilon_{w \mathrm{I}} N_{\mathrm{I}}^{25}+\epsilon_{w \mathrm{II}} N_{\mathrm{II}}{ }^{25}\right)
\end{aligned}
$$

where $F_{J}=\bar{\phi}_{J} T_{E} / S$ for $J=\mathrm{I}$ or II, $F_{w}=\phi_{w} T_{E} / S$, and $S$ is the integrated source yield (neutrons) over the detector exposure period. Correspondingly, equation (2) becomes

$$
\begin{aligned}
\bar{\sigma}_{A} & \equiv \frac{N_{F \mathrm{I}}+N_{F \mathrm{II}}}{S\left(\epsilon_{\mathrm{I}} N_{\mathrm{I}}{ }^{25} F_{\mathrm{I}}+\epsilon_{\mathrm{II}} N_{\mathrm{II}}{ }^{25} F_{\mathrm{II}}\right)} \\
& =\bar{\sigma}+\frac{F_{w} \bar{\sigma}_{w}\left(\epsilon_{w \mathrm{I}} N_{\mathrm{I}}{ }^{25}+\epsilon_{w \mathrm{II}} N_{\mathrm{II}}{ }^{25}\right)}{\epsilon_{\mathrm{I}} N_{\mathrm{I}}{ }^{25} F_{\mathrm{I}}+\epsilon_{\mathrm{II}} N_{\mathrm{III}}{ }^{25} F_{\mathrm{II}}}
\end{aligned}
$$

Figure 6 is a plot of $\bar{\sigma}_{A}$ vs $\left(\epsilon_{\mathrm{I}} N_{\mathrm{I}}{ }^{25} F_{\mathrm{I}}+\epsilon_{\mathrm{II}} N_{\mathrm{II}}{ }^{25} F_{\mathrm{II}}\right)^{-1}$ and the effective $R_{D}{ }^{2}$. The three points are so nearly co-linear that no sophisticated optimization of weighting had to be coinsidered in determining the intercept. However, different weightings were investigated to see which choice of weighting would justify the least combined error. It was found that the combined error was not sensitive to the weight given the intermediate point. Inclusion of this point tended to reduce the random error but to increase the systematic uncertainty (because of a larger error in background pit discrimination for this run).

The computation of the error in $\bar{\sigma}$ from the many error contributions in the measured quantities is too tedious to present in complete detail, but some general remarks and an example will be given. The common error propagation formula

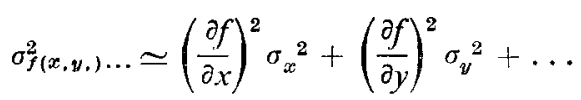

was used to combine the independent error contributions, both random and systematic. Correlated errors or covariance terms were avoided by breaking down each experimental error into its independent constituents. However, one significant approximation was made: all error sources were treated as symmetric (as likely to be positive as negative), whereas some error components are actually asymmetric. In addition to deriving a combined error estimate, separate subtotals of the systematic and random error categories were derived. All estimated errors are to be interpreted as one standard deviation.

The propagation of the uncertainty in the ${ }^{24} \mathrm{Na}$ half-life is particularly complicated because this factor affects the Mn bath comparison as well as the decay of the source between the fission measurement and calibration in the bath. In this case the sensitivity of the final $\bar{\sigma}$ value to the ${ }^{24} \mathrm{Na}$ half-life was computed by consistently assuming two separate values for the half-life throughout the data analysis and computing the derivative numerically. The assumed half life of $(15.00 \pm 0.03)$ hours (Merritt, 1971 ; Emery, 1972) leads to a $\pm 0.57 \%$ uncertainty in the final $\bar{\sigma}$ value.

The results of the data analysis and error propagation are presented in Fig. 6. The value derived

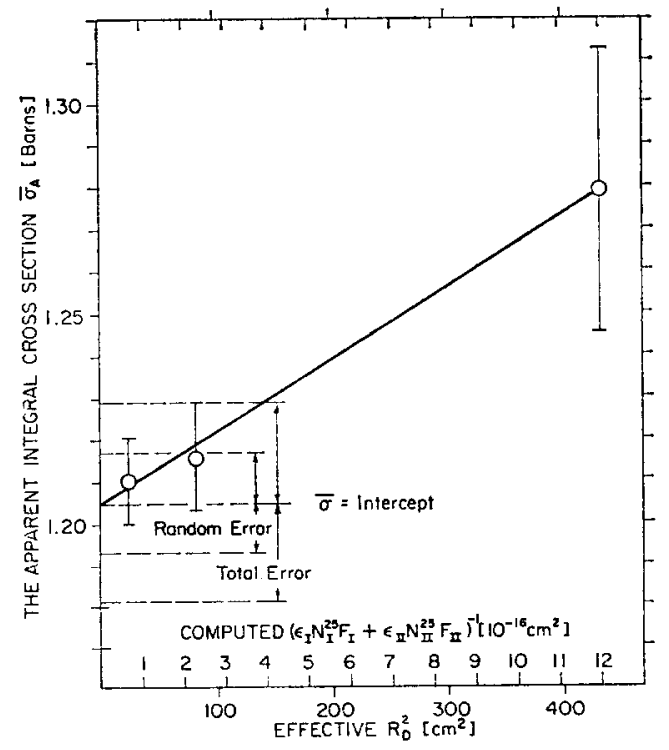

Fig. 6. The Apparent Integral Cross Section shows the effect of room-scattered neutrons. The extrapolation to zero source-detector spacing $\left(R_{D}=0\right)$ corrects for this effect. The error bars on the three experimental points show only random error magnitudes. Both random and total error magnitudes are indicated for the corrected result, $\bar{\sigma}$. 
Table 4.

\begin{tabular}{|c|c|}
\hline $\begin{array}{l}\text { Energy } \\
\text { (Upper end of } \\
\text { Interval) }\end{array}$ & $\left(\sec ^{-1} \mathrm{keV}^{-1} \times 10^{-3}\right)$ \\
\hline $\begin{array}{r}1000 \\
980 \\
960 \\
940 \\
920\end{array}$ & $\begin{array}{c}1.376 \\
28.45 \\
9.002 \\
1.238 \\
1.073\end{array}$ \\
\hline $\begin{array}{l}900 \\
880 \\
860 \\
840 \\
820\end{array}$ & $\begin{array}{l}0.8534 \\
0.7215 \\
0.7561 \\
0.6407 \\
0.6091\end{array}$ \\
\hline $\begin{array}{l}800 \\
780 \\
760 \\
740 \\
720\end{array}$ & $\begin{array}{l}0.5457 \\
0.4715 \\
0.4684 \\
0.3939 \\
0.5012\end{array}$ \\
\hline $\begin{array}{l}700 \\
680 \\
660 \\
640 \\
620\end{array}$ & $\begin{array}{l}0.3932 \\
0.4689 \\
0.3872 \\
0.3528 \\
0.2178\end{array}$ \\
\hline $\begin{array}{l}600 \\
580 \\
560 \\
540 \\
520\end{array}$ & $\begin{array}{l}0.1190 \\
0.1630 \\
0.1446 \\
0.08904 \\
0.08498\end{array}$ \\
\hline 500 & 0.01910 \\
\hline
\end{tabular}

Note: All energy Intervals are of $20 \mathrm{keV}$ width except the lowest, which is

$500 \mathrm{keV}$ wide. The $Q(E)$ distribution is normalized to 1 neutron/sec total

enission.

for the photoneutron spectrum-averaged cross section is $1 \cdot 20_{6}$ barns $\pm 2.04 \%$.

This spectrum-averaged result is mildly dependent on the particular photoneutron source construction, but is very nearly equal to the cross section at the median energy of the distribution since the cross section does not vary markedly over the breadth of the spectrum $(500-1000 \mathrm{keV})$. It is possible to express the result in terms of the cross section at a single energy point without significant increase in the overall uncertainty by use of relative cross section shape data of only moderate accuracy together with the computed energy distribution of the photoneutrons.

The computed energy spectrum from the Vesely Monte Carlo code is shown in Fig. 7 and tabulated in Table 4. The tail of neutrons of degraded energy is produced by neutron scattering and gamma (Compton) scattering within the source. The correction factor from the integral result to the value at $964 \mathrm{keV}$ was found to vary from 1.0034 to 1.0076 depending on the relative cross section data employed. A weighted average of $1.0045 \pm 0.0020$ was chosen, relying most heavily on data from the reports KFK-120 (Schmidt, 1962) and LA-3527 (Berlijn, 1968). In making this small correction, no dependence on the absolute normalization of

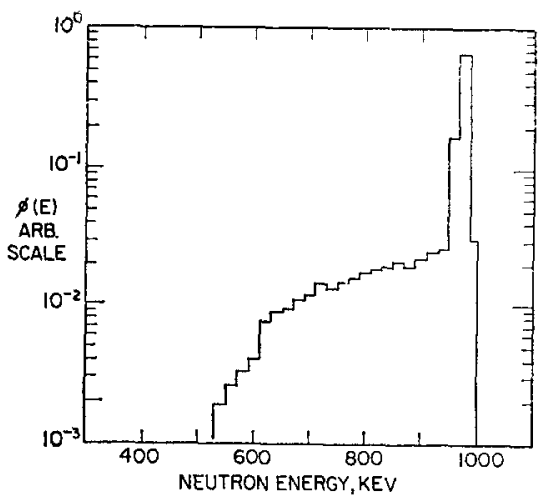

Fig. 7. The calculated neutron energy distribution from the $\mathrm{Na}-\mathrm{Be}$ source. The median energy of the distribution is $964 \mathrm{keV}$. Neutron scattering (particularly in the $\mathrm{Be}$ shell) and Compton scattering prior to the $(\gamma, n)$ reaction produce the tail of the distribution. About $22 \%$ of the neutrons have energies below $940 \mathrm{keV}$. The spectrum calculation was done by the Monte Carlo method using a code written by Vesely (See Bensch and Vesely (1969)).

other cross section data is incurred, because only the ratio $\sigma_{f}(E) / \sigma_{f}(964 \mathrm{keV})$ is used.

\section{RESULTS AND DISCUSSION}

The correction for the energy distribution of the neutrons allows expression of the measured integral 
Table 5.

\begin{tabular}{|c|c|}
\hline Experimental Factor & $\begin{array}{l}\text { Resulting Uncertainty } \\
\text { In Efnal Result } \\
\text { (\%) }\end{array}$ \\
\hline Scanner Bias and Background Discrimination ${ }^{a}$ & 1.1 .2 \\
\hline Angular Distribution of Fragment Emission & 0.92 \\
\hline Manganese Bath Comparisons of Sources & 0.42 \\
\hline NBS-II Reférence Source & 0.50 \\
\hline Neutron Scattering in Platinum Backings & 0.21 \\
\hline Pissile Deposit Masses & 0.50 \\
\hline $\begin{array}{l}\text { Propagated Errors In Net }{ }^{b} \text { Fission Counts I } \\
\text { Source Neutron }\end{array}$ & 0.82 \\
\hline $\begin{array}{l}\text { Propagated Errors in Source-Detector Spacing, } \\
\text { Neutron Angular Distribution, and Aperture } \\
\text { Geonetry }\end{array}$ & 0.60 \\
\hline $24 \mathrm{Na}$ half-life & 0.57 \\
\hline Energy Distribution of Source Neutrons & 0.20 \\
\hline Corbined Exror & 2.05 \\
\hline
\end{tabular}

The background pit discrimination error was $1.38 \%$ for the run at $9.2 \mathrm{~cm}$ spacing. The weighting of the $9.2 \mathrm{~cm}$ rua is discussed near the end of sect1on 3.4 .

bhe normalized fission count at wide source-detector spacing is subtracted from the normalized count at close spacting.

cross section in terms of a value at a specific neutron energy:

$$
\sigma_{f}(964 \mathrm{keV})=1.21 \pm 0.025 \mathrm{~b} .
$$

The estimated error of $0.025 \mathrm{~b}$ or $2.05 \%$ can be broken into two components: $1.78 \%$ systematic and $1.02 \%$ random (both standard deviations). A summary of the major uncertainties contributing to the combined error is given in Table 5 .

In Table 6 and Fig. 8, the result of the present work is compared with measurements listed in the SCISRS file of the BNL Neutron Cross Section Center. The present work agrees within $1 \%$ with measurements completed during the last ten years by Poenitz, Kaeppeler, Gayther, Cramer, Szabo and White. Except for the value reported by Szabo in 1970 , these recent measurements all give values slightly higher than that of the present work. Preliminary data given by Szabo at the 1972 Vienna Panel Meeting were lower than the present result by $2.5 \%$ or more.

An indirect comparison may also be made with a measurement of the fission-spectrum-averaged cross section of ${ }^{235} \mathrm{U}$. If the ENDF/B-III cross section value at $964 \mathrm{keV}(1.224 \mathrm{~b})$ is normalized according to the measured integral cross section $\bar{\sigma}_{f}\left(\chi_{c f}\right)$ value of Grundl, Spiegel and Eisenhauer (1972) (1207 \pm $52 \mathrm{mb}$ measured, $1241 \mathrm{mb}$ calculated from ENDF/ B-III data), the normalized value at $964 \mathrm{keV}$ is $1.19 \mathrm{~b}$. The normalized value is $1.6 \%$ below the present result and well within the error bars of both measurements.

The close agreement of the recent measurements in the range $950-1000 \mathrm{keV}$ is rather remarkable in

Table 6.

\begin{tabular}{|c|c|c|c|c|c|c|c|}
\hline First Author & $\begin{array}{l}\text { Nominal } \\
\text { Energy } \\
\text { (kev) }\end{array}$ & $\begin{array}{l}\text { Energy } \\
\text { Resolution } \\
\text { (kev) }\end{array}$ & $\begin{array}{l}\text { Reported or } \\
\text { Interpolated } \mathrm{b}, \mathrm{d} \\
\sigma(\mathrm{n}, \mathrm{f}) \\
\text { (barns) }\end{array}$ & $\begin{array}{l}\text { Quoted } \\
\text { Error } \\
\text { (x) }\end{array}$ & $\begin{array}{l}\text { Reference } \\
\text { (CSISRS } \\
\text { Data Set No.) }\end{array}$ & Date & $\begin{array}{c}\text { Comparison with } \\
\text { Present Work } \\
\text { (z) }\end{array}$ \\
\hline W. P. Poenitz & $965^{b}$ & $30^{\mathrm{c}}$ & $1.21^{\mathrm{b}}$ & 3.0 & $\begin{array}{l}\text { Nuc. Sc1. and Eng. 53, } 370 \\
\text { (10333) }\end{array}$ & 74 & 0.1 \\
\hline F. Xaeppeler & 966 & 21 & $1.2 I_{3}$ & 2.9 & $\begin{array}{l}\text { IAEA Panel, Vienna } \\
(90018 ; 2,3)\end{array}$ & 72 & 0.2 \\
\hline D. B. Gayther & 950 & 50 & $1.21_{8}$ & 4.0 & $\begin{array}{l}\text { AERE PR/NP, } 19 \\
(90017,2)\end{array}$ & 72 & 0.7 \\
\hline J. D. Cramer & 963 & $24^{c}$ & $1.22_{1}$ & 4.5 & $\begin{array}{l}I A-4420,45 \\
(10057,2)\end{array}$ & 70 & 0.9 \\
\hline I. Szabo & 1010 & 40 & $1.20_{5}$ & 2.9 & $\begin{array}{l}\text { Argonne CONF-701002,257 } \\
(90019,2)\end{array}$ & 70 & -0.4 \\
\hline P. H. White & 1000 & 50 & 1.22 & 2.5 & $\begin{array}{l}\text { 3. Nuc. En., 19, } 325 \\
(60441,4)\end{array}$ & 65 & 0.8 \\
\hline G. N. Snirenken & $965^{d}$ & & $1.19^{d}$ & -- & $\begin{array}{l}\text { Atomn. En. } 13,366 \\
(80058,9)\end{array}$ & 62 & -1.7 \\
\hline W. D. Allen & 1000 & -- & 1.22 & 1.6 & $\begin{array}{l}\text { Proc. Phys. Soc.;A70,573 } \\
(60808,11)\end{array}$ & 57 & 0.8 \\
\hline B. C. Diven & 944 & 39 & 1.27 & 3.9 & $\begin{array}{l}\text { Phys. Rev., 105, } 1350 \\
(51233,2)\end{array}$ & 57 & 5.0 \\
\hline R. L. Henkel. & 960 & -- & 1.26 & -- & $\begin{array}{l}\text { LA- } 2122 \\
(50520,10)\end{array}$ & 57 & 4.1 \\
\hline Present Work & 964 & 32 & 1.21 & 2.1 & (10314) & $-\infty$ & $-\infty$ \\
\hline
\end{tabular}

a (Value from other work -1.21 b)/1.21 b $\times 100 \%$.

bInterpolated from Poenitz's reported values of $1214 \mathrm{mb}$ at $950 \mathrm{keV}, 1209 \mathrm{mb}$ at $980 \mathrm{keV}$.

cspacing of data points.

dinterpolated from Smirenken's reported values 1.18 b at $950 \mathrm{keV}, 1.21 \mathrm{~b}$ at $1000 \mathrm{keV}$. 


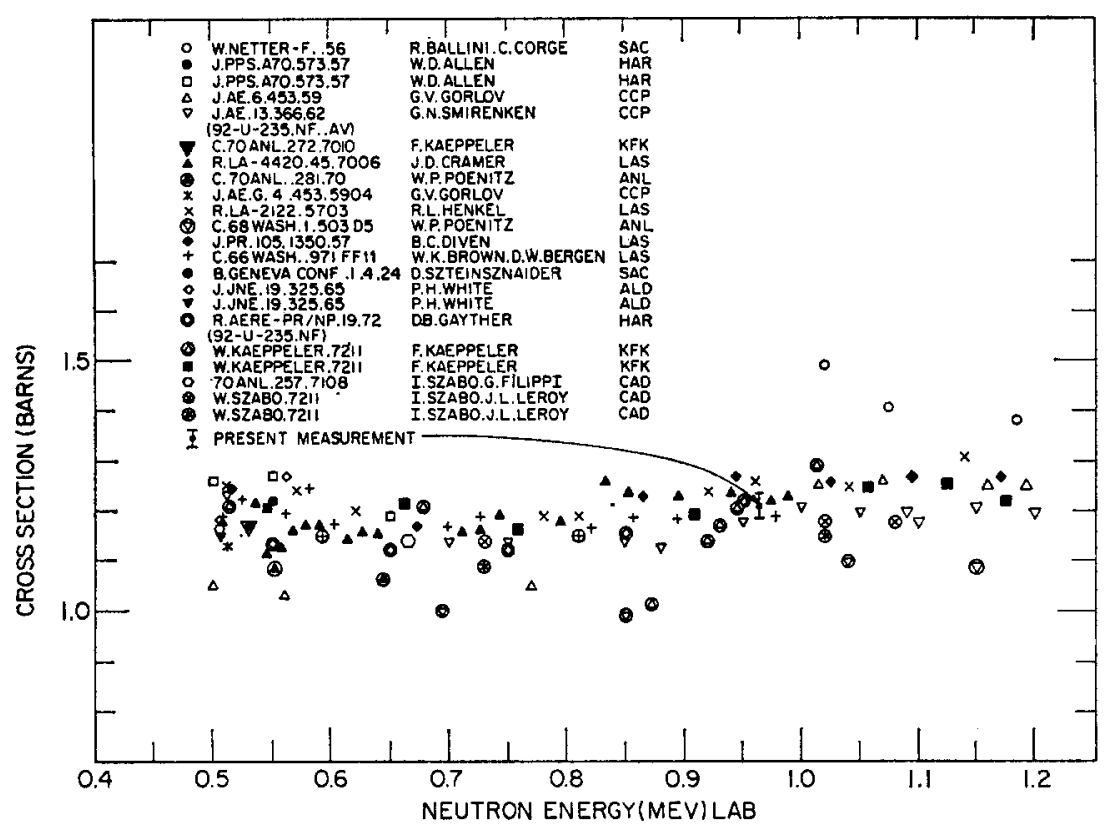

Fig. 8. Comparison with other measurements. The present result is added to the BNL plot of CSISRS data.

that the quoted errors are mostly in excess of $2.5 \%$. Significant discrepancies still persist over most of the energy range of interest for reactor calculations.

Acknowledgements-The authors wish to acknowledge the substantial assistance provided by William Stephany in the development of portions of the experimental facilities used in these measurements. Also, the analysis and counting of fission tracks was largely carried out by Katherine Fleming and her painstaking efforts deserve our sincere thanks.

\section{REFERENCES}

Allen W. D. and Ferguson A. T. G. (1957) Proc. phys. Soc. Lond. A70, 573. BNL NNCSC CSISRS Acc. No. $60808,10,11$.

Axton E. J., Cross P., and Robertson J. C. (1965) J. nucl. Energy A/B 19, 409.

Ballini R., Corge C., Julien J., and Netter F. (1961) Report $C E A-l 9 l 3$. BNL NNCSC CSISRS Acc. No. $60584,2$.

Bensch F. and Vesely F. (1969) J. nucl. Energy A/B 23, 537.

Benton E. V. and Nix W. D. (1969) Nucl. Instrum. Method 67, 343.

Berlijn J.-J. H., Hunter A. E., and Cremer C. C. (1968) Report $L A-3527$.

Brown W. K., Bergen D. W., and Cramer J. D. (1966) Conference on Neutron Cross Sections and Technology 1st Washington CONF 660303, 971. BNL NNCSC CSISRS Acc. No. 52040, 2.

Corman E. G., Jewell R. W., John W., Sherwood J. E., and White D. (1964) Phys. Lett. 10, 116.

Cramer J. D. (1970) Report $L A-4420,45$. BNL NNCSC CSISRS Acc. No. 10057, 2.

DeVolpi A. (1969) Metrologia 5, 128.
DeVolpi A. (1971) J. Nucl. Energy 24, 577.

Diven B. C. (1957) Phys. Rev. 105, 1350. BNL NNCSC CSISRS Acc. No. 51233, 2.

Emery J. F., Reynolds S. A., Wyatt E. I., and Gleason G. I. (1972) Nucl. Sci. Engng 48, 319.

Gayther D. B., Boyce D. A., and Brisland J. B. (1972) Report $A E R E-P R / N P, 19$. BNL NNCSC CSISRS Acc. No. 90017, 3.

Gilliam D. M. and Knoll G. F. (1970) Trans. Am. nucl. Soc. 13, 526.

Gorlov G. V., Gokhberg B. M., Morozov V. M., Otroshchenko G. A., and Shigin V. A. (1959) Atomn. Energy 6, 453. BNL NNCSC CSISRS Acc. Nos. 80033,9 and 40055, 2.

Grundl J. A., Spiegel V. Jr., and Eisenhauer C. (1972) Trans. Am. nucl. Soc. 15, 945.

Henkel R. L. (1957) Report $L A-2 l 22$. BNL NNCSC CSISRS Acc. No. 50520, 10.

Huynh V. D., Lafaye L., and Bréonce P. (1970) ProcèsVerbaux Comité International des Poids et Mesures 38, 76 .

Kaeppeler F. (1971) Neutron Standards and Flux Normalization. AEC Symposium Series 23 (Argonne 1970), 272.

Kaeppeler F. (1972) IAEA Panel on Neutron Standards and Reference Data Vienna (to be published). BNL NNCSC CSISRS Acc. No. 90018, 2, 3.

Merritt J. S. and Taylor J. G. V. (1971) Progress Report AECL-3912, 43.

Nesterov V. G., Smirenken G. N., and Shlah D. L. (1965) Report LA TR 66-41, FEI-33.

Noyce R. H., Mosburg E. R. Jr., Garfinkel S. B., and Caswell R. S. (1963) J. nucl. Energy A/B 17, 313.

Peakall K. A. and Antill J. E. (1960) J. nucl. Mater. 2, 194. 
Poenitz W. P. (1968) Conference on Neutron Cross Sections and Technology 2nd Washington NBS Special Publication 299, 503.

Poenitz W. P. (1971) Neutron Standards and Flux Normalization AEC Symposium Series 23 (Argonne 1970), 281. BNL NNCSC CSISRS Acc. No. 10296, 2.

Poenitz W. P. (1974) Nucl. Sci. Engng 53, 370. BNL NNCSC CSISRS Acc. No. 10333.

Rodriquez de Sastre M., Philippot J., and Moreau C. (1967) Report CEA-R 3218.

Ryves T. B. (1964) J. nucl. Energy A/B 18, 49.

Schmidt J. J. (1962) Report KFK-120 (EANDC-E-35 U). Scott K. T. and Harrison K. T. (1963) J. nucl. Mater. $8,307$.

Simmons J. E. and Henkel R. L. (1960) Phys. Rev. $120,198$.

Smirenken G. N., Nesterov B. G., and Bondarenko I. I. (1962) Atomn. Energy 13, 366. BNL NNCSC CSISRS Acc. No. 80058, 9.
Spiegel V. Jr. (1972) National Bureau of Standards, Private Communication.

Szabo I., Filippi G., Huet T. L., Leroy J. L., and Marquette J. P. (1971) Neutron Standards and Flux Normalization. AEC Symposium Series 23 (Argonne 1970), 257. BNL NNCSC CSISRS Acc. No. 90019, 2.

Szabo I., Leroy J. L., and Marquette J. P. (1972) IAEA Panel on Neutron Standards and Reference Data Vienna (to be published). BNL NNCSC CSISRS Acc. No. $90027,2,3$.

Szteinsznaider D., Naggiar V., and Netter F. (1955) Proceedings of the International Conference on the Peaceful Uses of Atomic Energy Geneva 4, 245. BNL NNCSC CSISRS Acc. No. 60319, 2.

Wallace P. R. and LeCaine J. (1943) Canadian NRC Document $M T-12$.

White P. H. (1965) J. nucl. Energy A/B 19, 325. BNL NNCSC CSISRS Acc. No. 60441, 4, 6. 